\title{
Reino y reinado de Dios en el Antiguo Testamento
}

El anuncio e inauguración del reinado de Dios constituye sin duda alguna el núcleo de la predicación de Jesús, la trama de toda su actividad mesiánica, el mensaje central de quien inició su misión predicando la definitiva cercanía de aquel reinado ( $\mathrm{Mc} 1,14 \mathrm{~s}=\mathrm{Mt} 4,17$ ), convencido de que «para esto» salió (Mc 1,38) y fue enviado ( $\operatorname{lc} 4,43)$. Se comprende, pues, que en el contexto de la oración enseñada por Él a sus discípulos $(\mathrm{Mt} \mathrm{6,9-13=Lc} \mathrm{11,2-4),} \mathrm{la} \mathrm{súplica}$ por la «venida» del reinado de Dios (Mt 6,10 = Lc 11,2) ocupe «un puesto central» '. ¿Es esa temática un mensaje nuevo? ¿Hunde, más bien sus raíces en la respectiva concepción veterotestamentaria?

\section{La importancia del tema}

El AT se hace reiteradamente eco de la temática sobre el «reinado» ${ }^{2}$ del «Rey» ${ }^{3}$ Jahveh, quien, con su «cetro» ${ }^{4}$ poderoso, «reina» ${ }^{5} \mathrm{y}$ «reinará» ${ }^{6}$ so-

1. S. SAbugal, El Padrenuestro en la interpretación catequética antigua y moderna, Salamanca 1980, 20-23.204-15: 22s. A este respecto, cf.: E. LOHMEYER, Das Vater-Unser (Abh. ThANT 23), Zürich ${ }^{3} 1952$, 59-75; N. PERrIN, The Kingdom of God in the teaching of Jesus, London 1963, 192s; J. Jeremias, Das Vater-Unser, Stuttgart 1967, 20s; ID., Neutestamentlische Theologie, Gütersloh 1971, 192s (trad. españ., Salamanca 1974, 232-34); J. ScHLossER, Le Règne de Dieu dans les dites de Jésus, I, Paris 1980, 246-284.

2. Sal 22,29; 103,19; 145,12.13; Abd 21; 1Cron 28,5; 29,11; Tob 13,1; Dan 3,33; 4,31; Sab 6,$4 ; 10,10$.

3. Num 23,21 ; Dt 33,5 ; 1 Sam 12,12 ; Is 6,$5 ; 33,22 ; 41,21 ; 43,15 ; 44,6$; Jer 8,$19 ; 10,7.10$; 46,18; 48,15; 51,57; Miq 2,13; Sof 3,15; Zac 14,9.16.17; Mal 1,14; Sal 5,3; 10,16; 24,7.8.9.10; 29,$10 ; 44,5 ; 47,3.7 .8 ; 48,3 ; 68,25 ; 74,12 ; 84,4 ; 95,3 ; 98,6 ; 99,4 ; 145,1 ; 149,2 ;$ Jdt 9,12; Tob 13,6.7.10.11.15,16; Est 4,17b.17f.17r; Dan 4,34; 2Mac 1,24; 7,9; 13,4; Ecclo 51,1.

4. Cf. Sal 45,7b; Miq 7,14; Ez 20,37; Est 4,17q.

5. 1 Sam 8,7 ; Is 52,7 ; Sal 47,$9 ; 93,1 ; 96,10 ; 97,1 ; 99,1 ; 146,10 ; 1$ Cron 16,31 (= Sal 96,10 ).

6. Ex 15,18; Is 24,23; Miq 4,7; Ez 20,33. 
bre su «trono» ${ }^{7}$ regio ${ }^{8}$. Es lo que, jubilosamente, confiesa la piedad israelita —de un modo especial- en los «salmos de entronización» ${ }^{9}$. Y tanto la fre-

7. $1 \operatorname{Re} 22,19$ (= 2Cron 18,18 ); $2 \operatorname{Re} 21,4$ (LXX: A); Is 6,$1 ; 66,1 ;$ Jer 3,$17 ; 14,21 ; 17,12$; 49,38; Bar 5,19; Ez 1,26; 10,1; 43,7; 1Cro 28,5; 29,23; 2Cro 9,8; Ecclo 1,8; Sal 45,7; 47,9; 89,15; 93,2; 97,2; 103,19; Dan 3,54 (LXX); 7,9; Sab 9,4.10.

8. Sobre el reinado de Dios en el AT, cf.: J. ORR, art. Kingdom of God, en: Hastings DB, II 844-56: 844-49; M.-J. LAGRANGE, La Règne de Dieu dans l'AT, en: RB 5(1908) 844-56: 844-49; J.-B. FreY, Le Royaume de Dieu, en: DB, V 1237-57: 1237-40; B. BARTMANn, Das Reich Gottes in der Heiligen Schrift (Bibl. Zeitfragen, 4-5), Münster 1912, 5-10; A. von GALL, Die Herkunft der Bezeichnung Jahwes als König (BZAW, 27), Giessen 1914, 145-60: 147-50; ID., Basileia tô Theoû, Heidelberg 1926, 41-42; O. EISSFELDT, Jahwe als König, en: ZAW 46(1928) 81-105 (= «Kleine Schriften», I 172-93; Cf. III 384s); H. GrESSMANN, Der Messias, Göttingen 1929, 25-44. 198-203. 209-20; G. von RAD, art. Basileus, en: ThWNT, I 562-69: 566ss; J, HERRMANN, art. cit., 81-84; J.A. OÑATE, El Reino de Dios en la Sagrada Escritura, en: EsBíbl 3(1944) 343-82: 344-51; ID., art. Reino de Dios, en: EncBib, VI 150-54: 150-52; H.-J. Kraus, Die Königsherrschft Gottes im AT (BHTh 13), Tübingen 1951; A. ALT, Gedanken über das Königtum Jahwes, en: «Kleine Schriften», I, München 1953, 345-57; J, BRIGHT, The Kingdom of God, New York 1952, 17-186; L. KÖHLER, Jahwä Malak, en: VT 3(1953) 188s; H. GROss, Die Weltherrschaft als religiöse Idee im AT (BBB, 6), Bonn, 1953; ID., Dominio universal de Dios según el AT, en: EncBib, II 990-94; M. García CoRdero, El reinado de Dios en el AT, en: CTom 81(1953) 3-33; ID., Teología de la Biblia, I, Madrid 1970, 559-69; II, Madrid 1972, 155-62; J. Ridderbos, Jahwäh Malak, en: VT 4(1954) 87-89; J. de FRAINE, L'aspect réligieuse de la royauté israelite (AnBib 3), Rome 1954, 117. 38. 458-61; M. BuBER, Königtum Gottes, Heidelberg ${ }^{3} 1956$; E. BEAUCHAMP, L'esperance du règne de Dieu et son developpement dans la trame de l'histoire d'Israel, en: VS 96(1957) 572-91; J. GRAY, The hebrew conception of the Kingship of God. Its origin and developpment, en: VT 6(1956) 268-85; ID., The Kingship of God in the Prophets and Psalms, en: VT 11(1961) 1- ; J. BonsiRven, o.c., 11-18; ID., Le Règne de Dieu suivant l'AT, en: «Mel. A. Robert», Paris 1957, 295-302; R. SCHNACKEMBURG, art. Basileia. I. Altes Testament, en: LThK, $\mathrm{II}^{2} 25$ s; ID., o.c., 1-22 (trad. españ., 3-30); ID., art. Reino de Dios, en: DTB, 888-907: 889-92; J. HEMPEL, art. Königtum Gottes im AT, en: RGG, III ${ }^{3}$ 1706-9; L. RosT, Königsherrschaft Jahwes in vorköniglicher Zeit?, en: ThLZ 85(1960) 721-24; V. MAGG, Malkût Jhwh, en: VTSuppl 7(1960) 129-53; W. EICHRODT, Theologie des $A T$, I 11s. 122-26 (trad. españ., 36s. 178-83); W.H. ScHMIDT, Königtum Gottes in Ugarit und Israel (BZAW, 80), Berlin 1961, 64-79; ID., Alttestamentlicher Glaube und seine Umwelt, Neukirchen-Vluyn 1968, 126-134; G.E. LADD, Jesus and the Kingdom, London 1966, 41-71; A. Gelston, Note on Jhwh mlk, en: VT 16(1966) 507-12. A. Schweitzer, Reich Gottes und Christentum, Tübingen 1967, 1-35; M. TREVES, The Reign of God in the OT, en: VT 19(1969) 230-43; R. Deville,-P. Grelot, art. Reino, en: VTB 762-67: 762-64; P. HÜnermanN, art. Reino de Dios, en: SM, V (Barcelona 1974) 880-97: 880-83; L. RUPERT, Jahwe der Herr und König, en: «Die Botschaft von Gott» (hrsg. v. K. Hemmerle), Freiburg 1974, 112-27; J. COPPENS, Le messianisme et sa rélève prophétique (BEThL, 38), Gembloux 1974, 7-30 (= Messianisme... prophétique); ID., La rélève apocalyptique du messianime royale. I. La royalité-Le régne-Le royaume de Dieu (BEThL, 50), Leuven 1979, 67-274 (= Royaute); J.A. SogGIN, art. Melek - Rey, en: DTMAT, I 1237-52: 1245-50; A. MATtioli, Dio e l'uomo nella Bibbia d'Israele, Roma 1981, 431-40; J. Coppens, art. Règne de Dieu, I, en: DBS, X (Paris 1981) 1-58 (bibliogr.: 57s).

9. Sal 47.93.96-99; Cf. a este respecto: A. von GALL, o.c., 148s; S. MowiNCKEL, Psalmestudien, II, Kristiana 1922, espec. 146-88; H. GREsSMANN, o.c., 212-18; A. FEuILlet, Les Psaumes eschatologiques du règne de Dieu, en: NRTh 73(1951) 244-60. 352-63; H.-J. KRAUS, o.c., 123-43; ID., Die Psalmen, I(BKAT, XV. 1), Neukirchen-Vluyn ${ }^{3} 1966,197-205$; H. Gross, o.c., («Die Weltherrschaft...»), 34-44; E. LEPINSKI, La royauté de Jahwé dans la poésie et le culte de l'Ancien Israel, Brussel 1965, espec., 91-335; J. GRAY, art. cit. (VT 6), 273-77; II., art. cit. (VT II), 1-29; R. 
cuencia y amplitud literaria reflejada en el uso de aquellos vocablos como la intensidad teológica de los mismos muestra, que la concepción sobre el reinado de Dios, muy generalizada en el antiguo oriente ${ }^{10} \mathrm{y}$ legado común de los pueblos semitas ${ }^{11}$, «ocupa un lugar importante en la religión de Israel» ${ }^{12}$, siendo tema central «de la fe y teología veterotestamentaria» ${ }^{13}$. No abordaremos aquí su génesis y desarrollo ${ }^{14}$, situándonos, más bien, al nivel redaccional de las fuentes literarias.

\section{El reinado presente de Dios}

«Jahveh es Rey» (Sal 10,16) y «reina» (Sal 93,1) «para siempre» (Sal $146,10)$. Estas y otras confesiones traducen la fe de Israel en el reinado presente de su Dios. Una fe, por lo demás enraizada en la experiencia históricosalvífica del «encuentro» con quien le sacó de Egipto y le introdujo en la «tierra», polarizada luego en diversos matices. Entre éstos destaca el que le confiesa como:

\section{1) El Rey celeste}

Así es designado «el Rey Jahveh Sabaoth» ${ }^{15}$, quien «asentó en los cielos su trono» (Sal 103,19; cf. Ecclo 1,8) celeste y «sagrado» (Sal 47,9), «fijado desde el principio» (Sal 93,2) y «eterno» en duración (Sal 95,7; Bar 5,19); un «trono excelso y elevado» (Is 6,1), majestuosamente fulgurante (Cf. Ez 1,26$28 ; 10-1)$ y glorioso (Sab 9,10), donde «se sienta como Rey eterno» (Sal 29,10)

SCHNACKENBURG, art. cit. (DTB), 890s; Id., o.c., 8-14 (trad. españ., 13-20); G. CASTELlino, Libro dei Salmi, Roma-Torino 1965, 615-17. 651-75; J.D. WATTS, Jahweh Malak Psalms, en: ThZ 21(1965) 341-48; F. AsENSIO, El Jahweh Malak de los «Salmos del Reino» en la historia de la salvación, en: EstBíb 25(1966) 299-315; J. CoPPENS, Les Psaumes de l'intronisation de Jahvé, en: EThL 42(1966) 225-31; ID., o.c., («Royauté»), 89-214; M. GarCía Cordero, o.c., II 158s; H. Gunkel, Die Psalmen, Göttingen ${ }^{5} 1968,201-4$. 419-31; ID., Einleitung in die Psalmen, Göttingen ${ }^{3}$ 1975,94,116; H.-J. Kraus, Theologie der Psalmen (BKAT, XV. 3), Neukirchen-Vluyn 1979, 105 112; J. COPPENS, art. cit., (DBS, X), 9-16.

10. Cf. O. EISSFELDT, art. cit., 85-88; M. BUBER, o.c., 39-50; W.-H. SCHMIDT, o.c., 17-63; J. ZANDEE, Gott ist König. Königssymbolismus in den antiken Gottesvorstellungen, besonders in der Religion des alten Aegypten, en: SHR 31(1975) 167-78.

11. O. EISSFELDT, art. cit., 84-87; W. EICHRODT, o.c., I 122 (trad. españ., I 178).

12. J. de FraINE, o.c., 461. Esa concepción es «el núcleo de la religión veterotestamentaria» (J. HeRrmAnN, art. cit., 81), siendo la realización del «Reinado universal de Dios el próton y el éschaton de Israel»: M. BUBER, o.c., LXIV.

13. J. COPPENS, Royauté, 273; Cf. también E. LEPINSKI, o.c., 457.

14. Una buena exposición de las diversas y dispares opiniones sobre el origen (religioso y temporal) de la fe israelítica en el reinado de Jahveh ofrece J. Coppens, Messianisme... prophétique, 19-24; ID., Royauté, 77-85.

15. Is 6,5 ; Jer 46,18; 48,15; 51,57; Zac 14,16.17; Cf. Mal 1,14. 
envuelto «de nube y densa bruma» (Sal 97,1-2a), y en el que, «sentado sobre querubines» (Sal 99,1) y rodeado de «todo el ejército celeste» $(1 \operatorname{Re} 22,19=$ 2 Cro 18,18 ) domina, «reina sobre las naciones» (Sal 47,9) como único soberano «de la tierra»y «de los dioses todos» (Sal 97,1.9), vestido por lo demás «de majestad y poder» (Sal 93,1-3) propios del «Rey de la gloria» (Sal 24,7-10. Tal es la dignidad regia, característica del Señor tres veces santo (Is 6,3) ${ }^{16}$, ante cuya presencia se estremece el cielo (Cf. Jc 5,5) y tiembla la tierra ${ }^{17}$, pues la «toca y se derrite» (Am 9,5), cuyo «incomunicable» y «misterioso» Nombre (Sab 14,21b; Jc 13,18) nadie puede conocer (Gen 32,30; Jc 13,17s), cuyo rostro no puede ser visto por ningún hombre sin que muera (Ex 33,20; Cf. 19,21), a la vista de cuya gloria el «hombre de labios impuros» se «siente perdido» (Is $6,5)$ y no puede menos de caer «rostro en tierra» $(\operatorname{Ez} 1,28 \mathrm{c}$; Dan 8,17$) \ldots$ No es ésa, sin embargo, la principal ni más antigua concepción veterotestamentaria sobre el señorío presente de Dios. Pues el trascendente Rey supramundano es, a la vez y ante todo, el inmanente monarca de esta tierra, reconocido y confesado por la fe y piedad del Pueblo elegido como su Rey ${ }^{18}$.

\section{2) El Rey de Israel}

El reiterado testimonio pre-monárquico acerca del reinado de Jahveh (Jc $8,22 \mathrm{~s} ; 1$ Sam $8,5.19 \mathrm{~s}$ ) muestra que, históricamente, aquella concepción no surgió durante el exilio ${ }^{19} \mathrm{ni}$ con la instauración de la monarquía ${ }^{20}$, sino más bien en el período de los Jueces y, probablemente, por influjo cananeo ${ }^{21}$. Al nivel de la actual redacción de las fuentes literarias, sin embargo, parece claro que la experiencia de Israel sobre el señorío regio de Jahveh es más antigua:

a) Se remonta a la epopeya salvífica del éxodo ${ }^{22}$. En efecto, el Dios que de-

16. Cf. Lev 19,$1 ; 20,7$. «El Santo de Israel»: Is 1,$4 ; 5,19.24 ; 10,17.20 ; 41,14.16$, etc.

17. Cf. Ex 19, 16-18; Jc 5,4; Sal 97, 5-6.

18. Cf. Ex 15,18 (= Is 43, 15-17); 19, 6-7; Num 23,21; Dt 33,5; Jc 8,23; 1Sam 8,7; 10,19; $12,12-25 ; 13,13-14 ; 15,26.35 ; 16,1-13 ; 2$ Sam 7, 12-16; 1Cron 28,5; 29,23; 2Cron 9,8; Sal 2, 6-8.

19. Así contra A. von GALl, (art. cit., 151; o.c., 41s) y otros autores citados por J. de FrAI$\mathrm{NE}$, o.c., 119-27; un origen post-monárquico postula G. von RAD, art. cit., 567s.

20. Contra O. EISSEFELDT, art. cit., 96.104s; A. ALT, art. cit., 349s; L. RosT, art. cit.; H.-J.Kraus, Psalmen, I 197s; J. GrüsSEMANn, Der Widerstand gegen das Königtum (WMANT, 49), Neukirchen-Vluyn 1978,78.82.

21. Así con: J. Gray, art. cit., (VT 6), 269-73; W.-H. SchMidT, o.c., 43-44. 70-72; P. GrELOT, Le sens chrétien de l'A T, Paris ${ }^{2} 1962$, 332, n. 3; F. STOLz, Strukturen und figuren im Kult von Jerusalem (BZAW, 118), Berlin 1970,179s; H. RINGGREN, Israelite Religion, 48; J. CoPPENS, Royauté, 267-69.

22. Cf.: H.W. WolfF, o.c., 168s; H.-J. KRAUS, Königsherrschaft..., 92-94; H. Gross, o.c. («Weltherreschaft...»), 23s; J. de Fraine, o.c. 127-34; M. BubER, o.c., 61-70.88. 103-11; J. BRIGTH, o.c., 19.28; ID., La historia de Israel, Bilbao 1966, 153-55; M. GARCÍA CORDERO, o.c., I 560; II 155s; J. Coppens, Messianisme... prophétique, 29; ID., Royauté, 267; ID., art. cit., (DBS, $\mathrm{X}), 21.50 \mathrm{~s}$. 
cidió liberar a «los hijos de Israel» de Egipto (Ex 3,7-20) por medio de Moisés (Ex 4,1-7,7) y, en una guerra santa (Cf. Ex 6,1; 7,3-5; 12,12) combatida mediante «toda suerte de prodigios» (Ex 7,8-12,34), los «sacó de la casa servil» (Ex 13,14.16), luego «iba al frente de ellos» (Ex 13,21) por el desierto como șu verdadero jefe, luchando (Ex 14,14.25) victoriosamente en el Mar Rojo, «al frente de! ejército de Israel» (Ex 14,19a) contra el faraón y su ejército (Ex 14,19b-30) ${ }^{23}$; una victoria, que Le valió la entusiasta aclamación del Pueblo definitıvamente salvado (Ex 15,1-21) como el triunfante "guerrero Jahveh» (Ex 15,3), quien, tras rescatarle y conducirle al lugar de su morada (Ex 15,13$17)$, «reinará por siempre» $($ Ex 15,18$){ }^{24}$. En esa gesta salvífica, por tanto, reconoció Israel en Jahveh a «su Rey» ${ }^{25}$.

b) Una fe confirmada y, en cierto modo, sellada luego por la alianza sinaítica (Ex 19,3-24,11). Antes de revelar a los liberados «hijos de Israel» (Ex 19,3-4) su voluntad, mediante las cláusulas de la alianza.o preceptos de la Ley (Ex 20,1-23,33), Jahveh condiciona («si») a la obediencia de éstos (Ex 19,5a) la reiterada promesa de ser aquéllos $S u$ «propiedad personal» así como $S u$ «reino de sacerdotes» y «nación santa» (Ex 19,5b-6a), galardonados asimismo con la bendición divina (Cf. Dt 11,26; Lev 26,3-12) así como con aquella paradisíaca comunión con Dios (Lev 26,12a; Cf. Gen 3,8a), cristalizada en el don de «la vida» (Dt 30,15.19). Lo que significa: Jahveh será el propietario de Israel, deviniendo éste su bendito y paradisíacamente feliz «reino sacerdotal»o dominio donde Él reina ${ }^{26}$, mediante el sacrificio de la obediencia a Su voluntad, por aquél ofrecido («jsacerdotes!») en la fidelidad a la alianza o fiel observancia de sus preceptos, lo que precisamente le constituye su «nación santa» o segregada de entre los pueblos y a Él exclusivamente consagrada. La reiterada aceptación de las cláusulas de la alianza (Ex 19,8a; 24,3,7), en el contexto de su ratificación solemne (Ex 24,3-11), significó la sumisa y libre «aceptación por parte de Israel de la Soberanía de Jahveh» ${ }^{27}$, autorreconociéndose como Su «reino sacerdotal» y reconociéndole «como su Rey» ${ }^{28}$. Aquélla fue, por tanto, una «alianza regia» ${ }^{29}$, de la que surgió «un tominio con un Señor y

23. Cf. J. Plastaras, i'he God of Exodus, Milwakee 1966, 165-201; R. de Vaus, Historia antigua de Israel, I, Madrid 1975, 369-70.437s; S. SABUGAL, Liberación..., 60-63.

24. Cf. M. BUBER, o.c, $107 \mathrm{~s}$.

25. Cf. Is 43, 15-17; Sal 74, 12-13.

26. Así con: P. van IMSCHOOT, Théologie de l'AT, I 246; M. BuBER, o.c., 104, n. 69.

27. J. BRIGHT, Historia..., 154 (Cf. 153-55); Cf. también: ID., o.c., 19.28; M. BUBER, o.c., 93-110: 98ss; W. EICHRODT, o.c., I 122 (trad. españ., 179); H. Gross, o.c, («Weltherrschaft...»), 23s; J. Plastaras, o.c., 214-16; J. CoPPENS, Messianisme... prophétique, 25s. 29; ID., Royauté, 265-70; R. de VAUX, o.c., I 399; J.A. SogGin, art. cit., 1247.

28. M. Buber, o.c., 115; J. Plastaras, o.c., 215; Cf. E. IACC', Théolcgie de l'A $A^{\top} .172$.

29. M. BUBER, o.c., 93-111. 
ullu subditos», flotando «desde entonces la idea del Reinado de Dios» ${ }^{\text {j0 }}$ sobre Israel.

c) Aquel reinado, en efecto, se prolonga durante los 40 años de peregrinación por el desierto, donde el jefe (o Rey) Jahveh «conduce» a Israel ${ }^{31}$ : Marchando «al frente de» él y combatiendo «por él» (Dt 1,30; 31,6.8). El «gran pecado» del becerro de oro (Ex 32,1-6.21.30), cometido por quienes exigieron «un dios que vaya delante de nosotros» (Ex 32,1), fue en realidad la primera apostasía del reinado de Jahveh sobre Israel, saldada luego por el «Dios misericordioso y clemente» (Ex 34,6) mediante la renovación de la alianza (Ex.34,10-27), solemnemente ratificada por el Pueblo tras la conquista y posesión de «la tierra» prometida (Jos 24,14-28), con la reiterada promesa de servir exclusivamente a Jahveh (Cf. vv. 14-24), su único Rey.

d) Una promesa no mantenida y sí repetidamente trasgredida durante el período de los jueces o carismáticos salvadores de Israel, suscitados por Jahveh (Jc 2,16.18): El Pueblo abandona reiteradamente el servicio exclusivo a su Dios, para «seguir a otros dioses» (Jc 2,17.19) y «servirles» ${ }^{32}$, cayendo entonces bajo la opresión de sus enemigos ${ }^{33}$, de la que le libra el juez que, conmovido «ante sus gemidos» ${ }^{34}$, les suscitaba Jahveh ${ }^{35}$, quien de este modo le salvaba «de la mano de sus enemigos» ${ }^{36}$. iDios era, pues, realmente su Rey ${ }^{37}$. Es lo que traduce, con claridad, la respuesta de Gedeón al requerimiento de «los hombres de Israel», quienes le piden reinar él y sus descendientes «sobre ellos», por haberles «salvado de» los madianitas $(\mathrm{Jc} 8,22)$ : «iNo reinaré yo ni mis descendientes sobre vosotros», sino que «Jahveh será vuestro Rey!» (Jc $8,23)$. Así rechazó enérgicamente el redactor deuteronomista la instauración de una monarquía cananea ${ }^{38}$, como lo hará luego al parodiar la unción regia de Abimelek en Siquem (Jc 9,1-20) ${ }^{39}$. Y si esos dos textos muestran «cuán vi-

30. W. Eichrodt, o.c., I 11s (urad. españ., 37); Cf. J. BRIGHT, Historia, 154.

31. Am 2,10; Cf. Dt 32, 11-12; Jos 24,17b.

32. Jc 3,6; Cf. 3,7.12; 4,1; 6,1, etc.

33. Cf. Jc $3,8.12-13 ; 4,2 ; 6,1 b-5$, etc.

34. Jc 2,18b; Cf. 3,9a.15a; 4,3a; 6,7.

35. Cf. Jc 2,18a; 3,9b; 4,6; 6, 7-14.

36. Jc $2,18 \mathrm{a}$; Cf. 7,7 .

37. Cf. 1Sam 10, 18-19; 12, 8-12.

38. Así con: J. Bright, Kingdom..., 32; H.-J. Kraus, Königsherrschaft, 92; J. de Fraine, o.c., 83s; M. Buber, o.c., 3-10; J.A. SogGin, Das Königtum in Israel (BZAW, 104), Berlin 1967, 15-20; F. GRÚSSEMANN, o.c., 42-54. Sobre Jc 8, 22-23, cf. también: W. BEYERLIN, Gechichte und heilsgeschichtliche Traditionsbildung im AT (Richter VI-VIII), en: VT 13(1963) 1-25: 19-23; G. HENTON, Judges VIII 22-23, en: VT 13(1963) 151-57; B. LINDARS, Gideon and Kingship, en: JThST 16(1965) 315-26.

39. Cf.: J. de Fraine, o.c., 83-85; A. Soggin, o.c., 20-25; H. Schmid, Die Herrschaft Abimelechs (Jdc 9), en: Jud 26(1970) 1-11; F. GRÜSSEMANN, o.c., 19-42. 
va estaba desde el principio en Israel la conciencia del reinado de Jahveh» ${ }^{40}$, su actual redacción literaria refleja el esfuerzo por superar la tentación de una nueva apostasía del Pueblo elegido contra el exclusivo reinado de su Dios.

e) Una tentación, por lo demás, no definitivamente soslayada. ¡Al contrario! La instauración de la monarquía ${ }^{41}$ significará precisamente sucumbir a ella. Es lo que refleja el relato sobre la elección de Saúl como primer rey de Israel (1Sam 8-12) ${ }^{42}$ : No pudiendo soportar el pésimo gobierno de los inicuos hijos de Samuel (1Sam 8,1-5), «los ancianos de Israel» exigen del anciano pro"eta darles «un rey, que nos juzgue como todas las naciones», de modo que (seamos como los demás pueblos» y «tengamos un rey, que.... vaya al frente de nosotros y combata nuestras guerras» (1Sam 8,5.19-20). ¡Un requerimienro grave! Porque si la petición de «un rey» significaba cometer el «gran mal» o pecado (1Sam 12,17.19) de rechazar el reinado sobre ellos de Jahveh y renegar a su verdadero Rey ${ }^{43}$, quien, marchando «al frente suyo» y combatiendo «por ellos» (Cf. supra), los libró «de los egipcios y de los reinos opresores» (1Sam 10,18), el deseo de ser «como» los demás pueblos y naciones implicaba aquella secularización institucional del Pueblo, que renuncia a ser el teocrático «Reino sacerdotal» de Jahveh (Cf. supra). Pero Dios obedece ( $j$ sic!) al pecado de Israel, ordenando a Samuel «escuchar su petición» y «darles un rey» (1Sam 8,9.22; Cf. Os 13,11), Saúl (1Sam 9,1-10.16): Elegido por Dios (1Sam 10,24; Cf. Dt 17,15) y proclamado rey «delante de Él» (1Sam 11,15), es Jah-

40. H. Gross, o.c., («Weltherrschaft...»), 25; así también: W. EICHrodT, o.c., I 123 (trad. españ., 179); J. BRight, Historia..., 181; K.H. BERnhardT, Das Problem der altorientalischen Königsideologie im AT (Suppl. VT, 8), Leiden 1961, 145s; J. LIVER, King, Kingship. In the Bible, en: EncJud, X (Jerusalem 1971), 1011-20: 1012; J. COPPENS, Royauté, 268.

41. Sobre la monarquía israleita, cf. G. von RAD, art. cit., 563s; ID., Das judäische Königsitual, en: «Gesam. Studien» (ThBücherei, 8), München 1958, $205-13$ (trad. españ., Salamanca 1976, 191-98); ID., Theologie des AT, I 318-40 (trad. españ., 381-433); M. NotH, Gott, König, Volk im AT, en: «Gesam. Studien» (ThBücherei, 6), München 1957, 188-229; R. PrEsS, Jahwe und sein Gesalbter, en: ThZ 13(1957) 321-34; G. BucEllati, Da Saul a Davide, en: BibOr 1(1959) 99-128; R. de VAUX, Instituciones del AT, Barcelona 1964, 141-68; ID., Le roi d'Israel vassal de Jahvé, en: Mél. E. Tisserant» (SteT, 23), Città del Vaticano 1964, 119-33; K.H. BERHARDT, o.c., espec., 91-290; J.A. Soggin, o.c., (supra, n. 38), 29ss; J. Liver, art. cit.

42. Cf.: J. de FrAINE, o.c., 75-117; M. BUBER, Die Erzählung vo Sauls Königswahl, en: VT 6(1956) 113-73; H. BILDBERGER, Samuel und die Entstehung desd insraelitischen Königtums, en: ThZ 13(1957) 442-79; W. BEYERLIN, Das Königscharisma bei Saul, en: ZAW 73(1961) 186-201; A. WEISER, Samuel. Seine geschichtleche Aufgabe un religiöse Bedeutung (FRLANT, 81), Göttingen 1962, 24ss; K.H BERNHARDT, o.c., 114-154; J.A. SogGIN, Charisma und Institution im Königtum Sauls, en: ZAW 75(1963) 54-65; ID., o.c., 29-57; G. von RAD, Theologie del AT, I 336-40 (trad. españ., 401-5); F. LANGLAMET, Les récits de l'institution de la royauté (ISam VII-VIII), en: RB 77(1970) 161-200 (bibliog.); F. GrüssemanN, o.c., 54-84; H.-J. Kraus, Theologie der Psalmen, 134-55.

43. 1Sam $8,7 b ; 10,18-19 ; 12,12$. 
veh quien lo «ha ungido», (ISam 15,17) y «establecido rey sobre» Israel (1Sam $12,13)$, con el encargo de que pueblo y rey Le sirvan «con todo su corazón» (1Sam 14,20-24) escuchando su voz y observando sus órdenes (1Sam 14,14-15), es decir, practicando fielmente la Ley de quien (Cf. Dt 17,18-19), de este modo, no renuncia y sí decide seguir siendo el verdadero Rey de Israel y de su rey. Así lo refleja su decisión de remover a Saúl del reino (1Sam 13,1314) y rechazarle como «rey de Israel» ${ }^{44}$, por la reiterada trasgresión de sus órdenes ${ }^{45} ;$ El Rey Jahveh no tolera la rivalidad del rey!, prefiriendo al sacrificio la obediencia (1Sam 15,22) o sumisión a su señorío. Ese tributo le rendirá precisamente David ${ }^{46}$, el «hombre según su corazón» (1Sam 13,14) y, por mandato suyo, ungido (1Sam 16,1-13) como «caudillo de su pueblo» (1Sam 13,14b), para sentarse «sobre el trono de» quien (Cf. 1Cro 29,23), en realidad, es el verdadero Rey de Israel. Nada de extraño, pues, si el monarca Le «consulta» antes de combatir contra los enemigos de Su pueblo ${ }^{47}$ y lucha contra éstos «en el Nombre de» Quien (1Sam 17,45), por ello le fortificó «para el combate» (2Sam 22,40$)$, haciéndole «triunfar en todas sus empresas» ${ }^{48}$ y salvándole «de todos sus enemigos» ${ }^{49}$, tras haberle prometido, en una alianza regia (2Sam 7,11-16) ${ }^{50}$, consolidar «el trono de su realeza» y asegurar «la permanencia eterna de su reinado ante» Él (2Sam 7,12.16). Dios es, por tanto, quien decide sobre el futuro de la dignidad regia y reinado del monarca, no siendo éste más que «el siervo» del «Señor Jahveh» ${ }^{51}$. Y eso será también Salomón ${ }^{52}$, el «siervo» de Jahveh $(1 \operatorname{Re} 3,7.8 .9)$, quien «le hizo rey» (1Re 3,7 a) y lo puso «sobre Su trono como rey de Jahveh» (2Cro 8,9), para que, sentándose «en el trono del reinado de Jahveh sobre Israel» $(1 \mathrm{Cro} 28,5)$, sucediese a David «como rey sobre el trono de Jahveh» (1Cro 29,23). El sabio y

44. 1Sam $15,11.23$ b. $26.35 ; 16,1 \mathrm{a}$.

45. 1Sam 13, 7b-12; 15,9.11.16-24.

46. Cf.: J. de Fraine. o.c., 112-15; S. Amsler, David, Roi et Messie, Neuchâtel 1963, 9-41;

47. Cf. 1Sam 23,$2 ; 30,7 ; 2$ Sam $5,19.23$.

48. 2Sam 8,6.14; Cf. 7,9a.

49. 2Sam 22,1.48.51; Cf. 7,9b.

50. Sobre la profecía de Natán (2Sam 7,1-17 = 1Cron 17, 1-15; Sal 89, 4-5.27-38), cf. H.-J. Kraus, Königsherrschaft..., 93; ID., Gottesdients im Israel, München ${ }^{2} 1962$, 222-34; L. RoST, Sinaibund und Davidsbund, en: ThLZ 72(1947) 129-34; S. HERRMANN, o.c., 92-103; A.H.J. GuNNEWEG, Sinaibund uns Davidsbund, en: VT 10(1960) 335-41; G. von RAD, Theologie des AT, I 320-31 (trad. españ., 384-95); R. de VAuX, art. cit., 124s; ID., Historia, I 399s; J. Coppens, Le Messianisme royal (Lect. Div. 54), Paris 1968, 39-52 (bibliogr.).

51. Cf. 2Sam 7,18-29; Sal 89,4.21: E. LEPINSKI, Le poème royale du Psaume 89, 1-5.20.38 (CahiersRB, 6), Paris 1967, 29-30.

52. Cf. F. Thierberger, Le Roi Salomon et son temps, Paris 1957; J. Bright, Historia..., 
prudente monarca fue, por tanto, esto: Vicegerente y representante del Rey Jahveh en el reinado del único reino de Israel. Y eso habrían sido sus descendientes, si la infidelidad de aquél a la regia alianza sinaítica (1Re $11,1-11 a)$ no hubiese motivado la decisión divina de quitarles una parte de su reino (1Re 11,11b-13).

f) Lo que tuvo lugar con el cisma político (1Re 12,20-25) y religioso (1 Re 12,26-33), tras el que inicia la sinuosa historia de ambos reinos, gobernados por sus respectivos monarcas (1Re 14-2Re 25). Deberán hacerlo, sin embargo, en aquella obediente sumisión a Jahveh prescrita por el deuteronómico «estatuto del rey» (Dt 17,14-20) ${ }^{53}$, aprendiendo así «a temer a Dios» (vv. 18-19) u obedecer y servir a Quien es, en rigor, el Rey del rey y el verdadero Soberano del Pueblo elegido. Es lo que, a lo largo de ese período, refleja el reiterado testimonio de los profetas, reconociendo en Jahveh al «Rey» (Jer 46,18; 48,15; 51,57 ) celeste (Is 6,5), universal (Jer 10,7) y eterno (Jer 10,10; Sal 10,16), Quien mora en Sión (Cf. Jer 8,19), «la ciudad del gran Rey» (Sal 48,3), y ha fijado en el Templo «el trono de su gloria» (Jer 14,21; 17,12). Los monarcas de Israel y de Judá deben ser, por tanto, Sus servidores y vasallos: Representantes del Rey Jahveh en el reinado de su Pueblo ${ }^{54}$. De ahí la valoración de aquéllos por el Deuteronomista según la fidelidad o rebelión a la regia alianza mosaica y davídica ${ }^{55}$. Por haber trasgredido ésta, en efecto, pereció el reino de Israel (Cf. 2Re 17,7-22), primero, y de Judá (Cf. 2Re 24,20-25,21; 2Cro 36,14-21), después. ¿Había el Rey Jahveh «rechazado a su ungido» y «desechado la alianza con su siervo»? (Sal 89,39s). En Su cólera desechó «a rey y sacerdote» (Lam 2,6c): ¿Habrá Jahveh abandonado a su «Reino sacerdotal?» ¿Habrá dejado de ser «Rey de Israel», Quien, sin embargo, «se sienta en Su trono eternamente» (Bar 3,3)?... Estos y otros interrogantes se formuló, desde la soledad y dolor del exilio ${ }^{56}$, el Pueblo elegido.

214-34; M. NotH, Historia de Israel, Barcelona, 1966, 193-204; J.A. SogGIN, o.c., 77-89; J. GoNZÁLEZ ECHEGARAY, art. Salomón, en: EncBib, VI 388-99

53. Para un análisıs de este deuteronomico "estatuto del rey», compuesto por el Deuteronomista en un esfuerzo por salvaguardar el carácter teocrático de la monarquía israelita, cf.: K. Gallin, Das Königsgesetz im Deuteronomium, en: ThLZ 76(1951) 133-38; J. de Fraine, o.c., 142-47; A. Clamer, Le Deutéronome (SB, II), Paris 1946, 625-28; G. von RAD, Das fünfte Buch Mose. Deuteronomium (ATD, 8), Göttingen 1964,85s.

54. Cf. J. de FraINE, o.c., 134-36; R. de VAUX, art. cit., 119-27; ID., Instituciones del AT, 148s; S. SABugal, Christós, Barcelona 1972, 17-19 (bibliogr.), y los trabajos previamente citados (n. 41).

55. Cf. G. von RAD, Theologie AT, I 346-59 (trad. españ. 412-26); ID., Die deuteronomistische Geschichtstheologie in den Königsbücher, en: «Gesam. Studien», 189-204: 197ss (trad. españ., 177-89: $183 \mathrm{ss}$ ).

56. Cf. Lam 1-5; Bar 1, 15-3,8. 
g) Y se habrían apagado, sin duda, en la desesperación, si los profetas -incansables «mensajeros de Dios» ${ }^{57}$ y de su Reinado (Cf. Is 52,7)—, no hubieran alzado entonces su voz: para asegurar, que Jahveh sigue siendo «el Rey de Jacob» y «de Israel» (Is 41,21; 44,6; Sof 3,15, etc.), anunciando su inminente reinado («iya reina tu Dios!») o la pronta salvación (Is 52,7 de Quien «es nuestro Rey» (Is 33,22), el Rey y Creador o Redentor de Israel (Is 43,15; 44,6); Él efectivamente «está en medio» del «Resto» de su Pueblo, perdonando y rescatándole «como un poderoso salvador» (Sof 3,12-15), dispuesto a «pasar delante de» él hacia su tierra (Miq 2,12s) para, tras realizar la gesta regia y salvífica del nuevo éxodo ${ }^{58}$, «reinar de nuevo» $(\mathrm{Ez} 20,33)$ y «por siempre» sobre Israel ${ }^{59}$. Un Reinado, por lo demás, ejercido mediante la fidelidad de éste a la interiorizada «Ley» (Jer 31,33a) de su Espíritu (Cf. Ez 11,19; $36,26 \mathrm{~s}$ ) o don de la «alianza nueva» (Jer 31,31; Ex 16,62) y «eterna» ${ }^{60}$, deviniendo de este modo Israel lo que antiguamente fue: Su Pueblo (Jer 31,33b; Ex 36,$28 ; 37,27 \mathrm{~s}$ ). No se detiene aquí, sin embargo, este profético anuncio del Señorío de Jahveh, ampliándose desde ahora, más bien, a más vastos horizontes de espacio y tiempo...

\section{El reinado escatológico y universal de Dios}

Aquel reinado de Dios, en efecto, no se circuhscribe al tiempo presente ni se limita al Pueblo elegido, proyectándose por el contrario hacia un futuro escatológico y alargándose hacia dominios universales: «En el monte Sión» ${ }^{61} \mathrm{y}$ desde el nuevo Templo, constituido «el lugar de Su trono» ${ }^{62}$, Dios reinará sobre la Idumea (Abd 21) así como sobre todas las naciones ${ }^{63}$ y sus dioses ${ }^{64}$, «sobre toda la tierra» ${ }^{65}$; pues «el Rey del cielo» ${ }^{66} \mathrm{y}$ «de toda la creación» ${ }^{67}$, de Quien «es cuanto hay en el cielo y en la tierra» (1Cro 29,1) por ser «el Rey del mundo» ${ }^{68}$ y el «único Rey» $(2 \mathrm{Mac} 1,24) \mathrm{u}$ «omnipotente Soberano de to-

57. 2Cron 36, 15-16; Is 44,26; Ag 1,13; Cf. Mal 3,1.

58. Cf. Ez 20, 33-35; Is 43, 15-21.

59. Cf. Ez 20,23; Miq 2, 12-13; 4,7; Is 43,15; 44,6; Sof 3, 15-17; Sal 47,7; 146,10.

60. Jer 32,$40 ;$ Ez 16,$60 ; 37,26$; Is 55,$3 ; 61,8$.

61. Is 24,23; Cf. Abd 21 .

62. Ez 43, 6-7; Cf. Jer 3,17; Zac 6,13a; Sal 47,9.

63. Cf. Sal 47,4.9; 96,10; Mal 1,14.

64. Sal 95,3 ; Cf. $97,1.9 \mathrm{c}$.

65. Sal 47,3.8; 97,1; 98, 4-6; Zac 14,9.

66. Tob 13,7.11.16; Dan 4,34.

67. Jdt 9,12; Cf. Sal 29,10; 96,10; 103,19b; Sab 12,16.

68. 2Mac 7,9; Cf. 7,23; 12,15; 13,14; 13,35. 
da potestad» ${ }^{69}$, ejerce «en Jacob juicio y justicia» (Sal 99,4), juzgando con equidad la tierra y los pueblos (Sal 98,9; Cf. 97,2) desde su eterno y «sagrado trono» (Sal 93,2; 47,9b), hasta que, tras haber sometido a las naciones rebeldes (Cf. Zac 14,1-3.16s) y haber «juzgado» a los soberbios reinos de la tierra (Cf. Dan 7,1-12), sea reconocido por aquéllas como «el Rey Yahveh Sebaoth» (Zac 14,16), es decir, el único «Rey sobre toda la tierra» (Zac 14,9; Cf. 2Mac 1,24).

\section{1) El reinado de Dios y del Mesías}

Esa sólida y arraigada fe en el reinado nacional y universal, presente y futuro de Dios traduce la jubilosa y exultante confesión: «iJahveh es Rey!» ${ }^{70}$, «ireina Jahveh!» ${ }^{71}$. Un reinado, por lo demás, realizado primero mediante el monarca israelita (Cf. supra) y, tras el ocaso de la monarquía, por Él ejercido mediante «Su siervo» el Mesías ${ }^{72}$.

a) El regio-davídico Rey futuro ${ }^{73}$, suscitado por Dios ${ }^{74} \mathrm{y}$, en virtud de la

69. 2Mac 3,24.29; Cf. 6,14.

70. Sal 93,1;96,10; Cf. 47, 2-3.7.

71. Sal 97,$1 ; 99,1 ;$ Cf. 47,9 .

72. Sobre la figura del Mesías veterotestamentario, en general, Cf.: A. GeLIN, DBS, V 1165-1212: 1170-1206; H. Gross, LThK, VII 336-9; J. OBERSTEINER, DTB 632-40; P.E. BONNARDP. Grelot, VTB 529-31; A. von Dall, o.c., 250-57; H. Gressmann, o.c., 220-80; R. Koch, Der Gesit und der Messias, en: Bib 27(1946) 241-68.376-403; A. BENTZEN, Messias, Moses redivivus, Menschenshn, (AbhThANT, 17, Zürich 1948; AA.VV., L'attente du Messie, Bruges 1954; J. KLAUSNER, The messianic idea in Israel, New York 1955, 26-271; S. HERRMANn, Die prophetische Heilserwartungen im AT (BWANT, 85), Stuttgart 1955; G. FOHRER, Messiasfrage und Bibelverständnis, Tübingen 1957, 11-22; H. RINGGREN, The Messias in the Old Testament (StBTh, 18), London 1956; AA.VV., CBQ 19(1957) 5-52; S. MowinCKel, He that Cometh, Oxford 1959, 125ss; M. WeISE, RGG, IV 902-4; O. EISSFELDT, RAC, II 1250-57; H. Gross, o.c., 75-110; ID., Der Messias im AT, en: "Bibel und zeitgemässer Glaube» (hrsg. von K. Schubert), Klosterneuburg 1965, 241-61; J. SCHARBERT, Der Messias im AT und im Judentum, en: «Die religiöse und theologische Bedeutung des AT», Würzburg 1967, 49-78; J. CoPPENS, Messianisme... prophétique, 31-153; ID., Messianisme, en: «Catholocisme», IX 9-19: 12-16; S. SABugal, Christós, Barcelona 1972, 15-25 (bibliogr.: XIIIs); H. CAZELles, El Mesías de la Biblia (trad. españ.), Barcelona 1981.

73. Am 9,11; Os 3,5; Is 4,2; 9, 5-6; 11, 1-11; Miq 5, 3-1; Jer 23, 5-6 (= 33, 15-16); Ez 34, 23-24; 37, 24-26; Zac 3,8; 6,12; 9, 9-10; Ag 2,23; Sal 2; 45; 72; 89; 110; Cf. H. GressMANN, o.c., 220-71; H.W. WolfF, art. cit., (ZAW 13), 171ss; L. Desnoyers, Les Psaumes, Paris 1925, 45-49; T. Orbiso, art. cit., 199-203; A. ColungA, El mesianismo en los Salmos regios, en: StudAnselm 27-28(1951) 208-30; A. GELIN, art. cit., 1177-92; H. RingGREN, König und Messias, en: ZAW 64(1952) 120-47; ID., o.c., 11-38; H.-J. KRAus, Königsherrschaft..., 90-99; ID., Psalmen, I LVIIILXX; H. Gross, o.c., («Weltherrschaft...»), 75-97; ID., art. cit., (supra, n. 72), 244-50; J.L. McKenzie, Royal messianism, en: CBQ 19(1957) 25-52; S. AMsler, o.c., (supra, n. 46), 43-66; J. SCHARBERT, o.c., 49-78; J. Coppens, Le Messie royale, en: NRTh 90(1968) 240-50; ID., Le messianisme royale (Lect. Div. 54), Paris 1968, 52-63 (bibliogr.); H. CA7El.les, o.c., 67-104.128-36.

74. Ez 34,23; Sal 89,20 . 
unción regia ${ }^{75}$, no sólo constituido hijo adoptivo suyo ${ }^{76}$ sino dotado con la plenitud de su Espíritu (Is 11,2), para practicar la «justicia» ${ }^{77}$ y, en calidad de «siervo» suyo ${ }^{78}$, reinar sobre su rescatado y reunido Pueblo ${ }^{79}$ así como sobre las naciones ${ }^{80}$, victorioso y humilde a la vez ${ }^{81}$, en una era de paz y santidad paradisíaca ${ }^{82}$;

b) El profético-mosaico «Siervo de Jahveh» ${ }^{83}$, elegido por Dios y dotado con su Espíritu (Is 42,1) no sólo para «ser alianza del Pueblo» (Is 42,6a) elegido, reconduciéndole a la tierra (Cf. Is 49,6a) tras haberle «abierto los ojos» ${ }^{84}$ y haberle liberado «de la cárcel» babilónica ${ }^{85}$, sino también para ser

75. Sal 45,$8 ; 2,2 ; 89,21.39$.

76. 2 Sam 7,14 ( = 1Cron 17,$13 ; 22,10 ; 28,6)$; Sal 2,7; 89,27s.

77. Is 11, 3-5; Jer 23,5s; Sal 72,4.7a. 12-14.

78. Ez 34,23s; 37,24s; Cf. Sal 89,4.21.40.

79. $\mathrm{Ez} 34,23 ; 37,24$.

80. Cf. Sal 2,8; 72, 8-11; 110,2; Zac 9,10.

81. Zac 9,9; Sal 2,8.

82. Cf. Is $11,6-9 ; \mathrm{Sal} 72,7 \mathrm{~b} ; \mathrm{Zac} 9,10$.

83. Is 42, 1-9; 49, 1-6; 50, 4-9; 52, 13-53, 12; Cf. (además de los comentarios a Isaías II: $\mathrm{CH}$. R. North, P.E. Bonnard, G.A.F. Kningth, C. Westermann, K. Elliger...): H. Gressmann, o.c., 287-339; J.S. van den PloEg, Les chants du Serviteur de Jahvé dans le second livre d'Isaïe, Paris 1936; A. BENTZEN, o.c., 42-71; C. LindHAgen, The Servant-motif in the OT. A priliminary study to the Ebed-Jahweh problem in Deutero-Isaiah, Uppsala 1950; J. LindBLOM, The ServantSongs in Deutero-Isaiah, Lund 1951; R.H. TouRNAY, Les chants du Serviteur dans la seconde partie d'Isaïe, en: RB 59(1952) 355-84. 481-512; 64(1957) 604-7; ID., art. Siervo de Jahvweh, en: EncBib, IV 666-69 (bibliogr.); H. Gross, o.c., 97-100; W. ZIMMERLI, art. Paîs Theou, en: ThWNT, V 653-76: 664ss; H. CAZELlES, Les poèmes du Serviteur, en: RSRel 43(1955) 5-55; ID., o.c., 108-113; C.R. NoRTH, The Suffering Servant in Deutero-Isaiah, Oxford ${ }^{2} 1956$; G. PIDOuX, Le Serviteur Souffrant d'Isaie 53, en: RThPh 6(1956) 36-46; A. GELIN, art. cit., 1192-96; S. MowINCKEL, o.c., 187-257; J. COPPENS, Les origines littéraires des poèmes du Serviteur de Jahvweh, en: Bib 40(1959) 248-58; ID., Le Serviteur de Jahweh: Vers la solution d'une énigme, en: SPag 1(1959) 334-54; J.E. Menard, Le titre de Païs Theoû, en: ib. 314-21; A.S. KAPELRUd, The identity of the Suffering Servant, en: «Near East Studies» (in honour of W.F. Albright), Garden City (N.Y.) 1961, 305-14; H. Gross, art. cit., 250-53; J. MoRGENSTERn, The Suffering Servant: $A$ new solution, en: VT 11(1961) 292-320. 406-31; ID., Two additional notes to "The Suffering Servant-A new solution», en: VT 13(1963) 321-32; C. CHAVASSE, The Suffering Servant and Moses, en: CQR 165(1964) 152-63; W.M.W. RoTH, The anointing of the Suffering Servant, e: JBL 83(1964) 17179; H.H. RowLEY, The Servant of the Lord and other essays, Oxford ${ }^{2} 1965$, 3-93; H.M. ORLINS$\mathrm{KY}$, The so-called «Servant of the Lord» and «Suffering Servant», en: «Studies on the second part of the Book os Isaiah» (Suppl. VT, 14), Leiden 1967, 1-133; ID., The so-called «Suffering Servant» in Isaiah 53, en: «Interpreting the Profetic Tradition» (ed. H.M. Orlinsky), New York 1969, 225-73; J.M. WARD, The Servant Songs in Isaiah, en: Rev Exp 65(1968) 133-46; J. CoPPENS, Messianisme... prophétique, 41-113; L.E. WILSHIRE, The Servant-City: A new interpretation of the «Servant of the Lord» in the Servant Songs of Deutero-Isaiah, en: JBL 94(1975) 356-67; C.G. KRUSE, the Servant Songs: Interpreting trends since C.R. North, en: StBTh 8(1978) 3-27; A. Gelin-L. Monloubou, El Mediador de la salvación: El Siervo, en: «Introducción a la Biblia», II (dirig. H. Cazelles), Barcelona 1981, 474-79; H. CAzelles, o.c., («El Mesías...»), 108-113; P. Grelot, Les Poèmes du Serviteur (Lect. Div., 103), Paris 1981, espec., 21-117.

84. Is 42,7.16. 18-19; 43,8: Cf. S. SABUgAL, La embajada mesiánica de Juan Bautista, Madrid 1980,175 , n. 199.

85. Is 42,7 ; Cf. 42,$22 ; 43,14 ; 45,2$. 
«luz de los gentiles» ${ }^{86}$, implantando «en la tierra el derecho» y «el juicio» salvífico (Is 42,4 ) de la gratuita liberación de Dios (Cf. Is 51,4-5), quien sobre su probado, paciente y confiado Siervo ${ }^{87}$ «descargó la culpa de todos» ${ }^{88}$, para ser por medio de él justificados todos (Is 53,11 ) y «llegar su salvación hasta los confines de la tierra» (Is 49,6c);

c) El mesiánico Hijo del hombre celeste ${ }^{89}$, a quien el divino «Anciano», tras juzgar y destruir los inicuos reinos de la tierra (Cf. Dan 7,1-12), otorgará el imperio sobre «todos los pueblos, naciones y lenguas» (Dan 7,13,14a), deviniendo el suyo «un imperio eterno», cuyo «reinado jamás será destruido» (Dan 7,14b).

El mesiánico Rey, Profeta e Hijo del hombre es, pues, instrumento salvífico de Dios o siervo suyo en la instauración de aquel reinado escatológico y universal, propio de quien, hacia el ocaso de la revelación veterotestamentaria, es confesado como «el Soberano» ${ }^{90}$, «el Rey único y bueno» (2Mac 1,24), «el Rey del mundo» $(2 \mathrm{Mac} 7,9)$ y «el Rey de reyes» $(2 \mathrm{Mac} 13,4)$, de quien éstos no son más que «ministros de su reinado» $(\mathrm{Sab} 6,4)$.

Resumiendo estos análisis podemos decir: que en el contexto de la temática central sobre el reinado de Dios, el AT subrayó ante todo la presencialidad del señorío celeste de Jahveh, quien, a raíz de la gesta salvífica del éxodo y con ocasión de la alianza sinaítica, devino Rey de Israel mediante la obediente sumisión de éste a Su voluntad; un señorío que, en reiteradas vicisitudes de fidelidad y rebelión, ejercerá sobre su «reino sacerdotal» mediante el vasallaje de jefes carismáticos, primero, y de reyes después; si se oscurecerá con el exilio babilónico, re-amanecerá con el nuevo éxodo, mediante la fidelidad del «Res-

86. Is 42,$6 ; 49,6 \mathrm{~b}$.

87. Cf. Is $50,4-9 ; 53,2-12$.

88. Is 53,6 ; Cf. $53,4-5.11-12$.

89. Cf.: A. BENTZEN, o.c., 72-74; T.W. MANSON, The Son of Man in Daniel, Enoch and the Gospels, en: BullJRIL 32(1949-50) 171-93: 173-75; H. GROss, o.c., 101-5; ID., art. cit., 253-56; L. Rost, Zur Deutung des Menschensonhes in dan 7, en: «Gott und die Götter» (FsE.Fascher), Berlin 1959, 41-43; K. KoCH, Die Weltreiche im Danielbuch, en: ThLZ 85(1960) 829-32; J. MUILENBURG, The Son Man in Daniel and in the Ethiopie Apocalypse of Enoch, en: JBL 79(1960) 197209; A.B. RHODES, The Kingdoms of Men and the Kingdom of Gog. A study of Daniel 7, 1-14, en: Interpr 15(1961) 411-30; J. MORGENSTERN, The «Son of Man» of Daniel 7,13f, en: JBL 80(1961) 65-77; J. CoPPENS, L'origine du symbole «Fils d'Homme», en: EThL 34(1963) 100-104; ID., La visión daniélique du Fils d'Homme, en: VT 19(1969) 171-82; C. COLPE, Ho huiós toû anthrópoû, en: ThWNT, VIII 403-81: 422-25; A. CAQUOT, Les quatre bêtes et le «Fils d'Homme», en: Sem 17(1967) 37-71; P. Grelot, L'intronisatio du Fils d'Homme, en: AS 65(1973) 30-35; J.J. Collins, The Son of Man and the Saint of the Most High in the Book of Daniel, en: JBL 93(1974) 50-66; J. COPPENS, Le Fils d'Homme vétéro- et intertestamentaire, Leuven 1982.

90. 2Mac 5,17; 6,14; 9,13; 15,22; «el gran Soberano»: 2Mac 5,20. 
to» liberado a la interiorizada Ley (;el Espíritu!) de la «nueva alianza»; un reinado, por lo demás, pre-anunciado ahora como escatológico y universal, que el Rey Jahveh ejercerá mediante el regio servicio del Mesías.

Santos SABUGAL, OSA 